\title{
Manifestaciones clínicas en la menopausia y sus modificaciones con la terapia hormonal de sustitución
}

\author{
Ensayo clínico multicéntrico colombiano comparativo de dos mezclas hormonales (estrógenos \\ conjugados más medroxiprogesterona vs. valerato de estradiol más acetato de ciproterona)
}

Fabio Sánchez E.*; Jaime Urdinola M.**; William Onatra H. ${ }^{* * *}$; Héctor Posso V.**** Jacinto Sánchez A.*****;
Rolf Alwers C.*****

\section{RESUMEN}

INTRODUCCION: La mujer en el climaterio presenta múltiples síntomas y signos como consecuencia del déficit estrogénico. Existen diversos medicamentos, esquemas y dosis utilizadas en la terapia hormonal de sustitución (THS). Se evalúa en el presente estudio la eficacia de dos diferentes esquemas terapéuticos combinados con estrógeno y gestágeno en mujeres perimenopáusicas.

MATERIALES Y METODOS: Se diseñó un ensayo clínico controlado, multicéntrico, aleatorizado, abierto y comparativo entre dos combinaciones hormonales diferentes (estrógenos conjugados $0.625 \mathrm{mg} /$ día más acetato de medroxiprogesterona $5 \mathrm{mg}$ del día 11 al 21 -grupo A- y valerato de estradiol $2 \mathrm{mg}$ /día por 21 días más acetato de ciproterona $1 \mathrm{mg}$ del día 11 al 21, grupo B.

Se reclutaron, entre abril de 1993 y diciembre de 1995, 104 mujeres voluntarias sanas, en perimenopausia y con presencia de oleadas de calor y otros síntomas secundarios al hipoestrogenismo. Se incluyen 87 mujeres que cumplen los requisitos para el presente análisis.

Las pacientes fueron seguidas clínicamente durante un año, con controles al comienzo, al mes, tercer, sexto, noveno y doce meses de tratamiento.

Se evaluaron las diferencias por grupo de tratamiento y en los diferentes tiempos. Para las variables cuantitativas se realizó el análisis de ANOVA y para las cualitativas el test de chi cuadrado o el de Fisher, usando como nivel de significancia una $p<0.05$.

RESULTADOS: Al término de un año los dos preparados hormonales redujeron la prevalencia de las oleadas de calor (grupo $\mathrm{A}=\mathbf{7 5 . 5} \%$, p $<\mathbf{0 , 0 0 0 1}$ y grupo $B=83.3 \%, p<0.00001$ ); la mayor reducción se produjo especialmente entre el primer y tercer mes de tratamiento.

La frecuencia de las oleadas de calor se redujo igualmente en ambos grupos de manera significativa (p<0.00001), especialmente para aquellos episodios clasificados como severos (más de cinco episodios por día), los cuales desaparecieron prácticamente desde el tercer mes de THS.

Los episodios de sudoración nocturna se redujeron significativamente en ambos grupos, en especial aquellos calificados como severos.

Hubo mejoría importante de síntomas como insomnio, fragmentación del sueño, manifestaciones depresivas, palpitaciones y dispareunia.

No hubo modificaciones en el índice de masa corporal ni complicaciones tromboembólicas.

Los efectos secundarios más frecuentes en ambos grupos fueron: tensión mamaria, náusea, edema y cefalea, más frecuentemente al comienzo de la THS.

CONCLUSIONES: En mujeres perimenopáusicas, la THS con dos diferentes preparados de estrógeno y gestágeno es bien tolerada e igualmente eficaz para el control de los principales síntomas climatéricos.

PALABRAS CLAVES: Menopausia, terapia hormonal de sustitución (THS), estrógenos conjugados, acetato de medroxiprogesterona, valerato de estradiol, acetato de ciproterona.

Trabajo distinguido con el Premio Institucional Dr. Fernando Cardona Arango, XX Congreso Colombiano de Obstetricia y Ginecología, Medellín, 1996. Este estudio contó con el apoyo económico de Química Schering Colombiana S.A. Santafé de Bogotá, D.C. Ensayo Clínico No. 92033.

Profesor Titular, Universidad de Antioquia. Programa de Reproducción - Clínica del Prado. Medellín.

Departamento de Ginecología, Obstetricia y Reproducción Humana. Fundación Santa Fe de Bogotá. Profesor Escuela Colombiana de Medicina.
*** Profesor Asociado. Departamento de Obstetricia y Ginecología. Facultad de Medicina, Universidad Nacional de Colombia. Clínica de Climaterio, Instituto Materno Infantil. Santafé de Bogotá.

**** Coordinador Unidad de Estudios Epidemiológicos. Instituto Nacional de Cancerología. Santafé de Bogotá.

***** Profesor Asociado. Departamento de Obstetricia y Ginecología. Facultad de Medicina, Universidad Nacional de Colombia. Clínica de Climaterio, Instituto Materno Infantil. Santafé de Bogotá.

****** Departamento de Medicina Interna. Hospital Santa Clara, Escuela Colombiana de Medicina. Asesor Médico. Química Schering Colombiana S.A. Santafé de Bogotá. 


\section{SUMMARY}

BACGROUND: Women in the menopause present multiple signs and symptoms as a consequence of estrogen deficiency.

There are several drug treatments and different recommended dosages for hormone-replacement therapy (HRT). We evaluated the efficacy of two different hormonal regimens with estrogen and progestogen in perimenopausal women.

METHODS: An open, multicentric, randomized clinical study was designed to compare two different combined hormonal preparations (conjugated estrogens $0,625 \mathrm{mg}$ daily for 21 days plus medroxyprogesterone acetate $5 \mathrm{mg}$ from days 11 through 21 - group A, and estradiol valerate $2 \mathrm{mg}$ daily for 21 days plus cyproterone acetate $1 \mathrm{mg}$ from days 11 through 21, group B). 104 healthy climacteric women with flashes, nocturnal sweats and other symptoms due to estrogen deficiency were recruited between april 1993 and december 1995 . We report on 87 women see for a period of one year with follow-up visits after recruitment and one, three, six, nine and twelve months of HRT.

Statistical analysis was performed to ascertain differences in proportions between groups and for changes during treatment time. We used ANOVA and chi-square or Fisher's test, with a level of significance $p<0,05$. All analyses were done using 6.01 (CDC, 1994).

RESULTS: After one year of hormone-replacement therapy with combined estrogen and progestogen, both preparations reduced the prevalence of hot flashes (group $A=75.5 \%, p<0.0001$, group $B=83.3 \%, p<0,00001$ ); the response to HRT was most pronounced during the first three months of treatment.

The frequency of hot flashes was also significantly reduced by both regimens $(p<0.00001)$, in particular for those reported as severe (more than 5 episodes per day), which almost disappeared after three months of therapy.

Episodes of night sweats were significantly reduced in both groups, and also specially those perceived by women as severe.

Important relief of symptoms like insomnia, sleep disturbances, depressive mood, palpitations and dyspareunia was also recorded.

There were no changes in body mass index and no thromboembolic disorders ocurred.

The most common side effects in both groups were breast tenderness, nausea, edema and headache, ocurring most commonly at the beginning of HRT.

CONCLUSIONS: In perimenopausal women, HRT with two different combined estrogen and progestogen preparations was well tolerated and showed equal efficacy for the relief of the most common climacteric symptoms.

KEY WORDS: Menopause, hormone-replacement therapy (HRT), conjugated estrogens, medroxyprogesterone acetate, estradiol valerate, cyproterone acetate.

\section{Introducción.}

La mujer durante el climaterio presenta múltiples síntomas como consecuencia del déficit estrogénico, entre los cuales se destacan las oleadas de calor, la sudoración diurna y nocturna, los trastornos del ánimo y del sueño, entre otros. Estas manifestaciones, si no son tratadas, pueden alterar su calidad de vida (1).

Un estudio reciente sobre la menopausia en Colombia (2) evaluó las principales manifestaciones clínicas, sin encontrar diferencias con los resultados publicados en la literatura mundial. Hasta la fecha, sin embargo, no se ha realizado un estudio comparativo de diferentes tratamientos hormonales sobre los síntomas del hipoestrogenismo en mujeres colombianas.

Son diversos los preparados, esquemas y dosis utilizadas en la terapia hormonal de sustitución THS, los cuales mejoran las diferentes manifestaciones clínicas que se presentan a corto y mediano plazo así como también pueden ejercer un efecto protector a largo plazo sobre el sistema cardiovascular y óseo (1-9).

La principal justificación del presente estudio fue comparar la eficacia de dos tipos de tratamiento, uno ampliamente utilizado desde hace varios años en el mundo (estrógenos conjugados más acetato de medroxiprogesterona) y otro que incluye un estrógeno (valerato de estradiol) extensamente utilizado en Euro$\mathrm{pa}$, asociado por primera vez a un gestágeno derivado también de la 17- $\alpha$ hidroxiprogesterona (acetato de ciproterona).

\section{Material y métodos}

Entre abril de 1993 y diciembre de 1995 se reclutaron 104 mujeres sanas perimenopáusicas con los siguientes criterios de inclusión: Presencia de oleadas de calor y otros síntomas secundarios al hipoestrogenismo, con útero y ovarios intactos y un índice de masa corporal (IMC) normal (19.1 - 25) quienes dieron su consentimiento escrito para participar en el estudio. Las pacientes fueron reclutadas en el Instituto Materno Infantil de Bogotá, Fundación Santa Fe de Bogotá y Clínica del Prado, en Medellín. El estudio fue aprobado por el Comité de Etica del Departamento de Obstetricia y Ginecología de la Universidad Nacional de Colombia y el Comité de Investigaciones y Publicaciones Biomédicas de la Fundación santa Fe de Bogotá.

Se excluyeron del estudio pacientes con citologías cérvicovaginales francamente anormales, enfermedad hepática aguda o crónica, antecedentes de ictericia idiopática del embarazo, síndrome de Dubin Johnson o Rotor, antecedente de enfermedad tromboembólica, anemia de células falciformes, presencia o sospecha de tumor maligno de útero, mamas, hígado o hipófisis, dislipidemias e hiperlipoproteinemia familiar, miomatosis, adenomiosis o endometriosis, hipertensión arterial, diabetes u otras enfermedades endocrinas, historia de herpes genital, otosclerosis, esclerosis múltiple, epilepsia, porfiria y administración de agentes hipolipemiantes, tiazidas y estrógenos, por medio de implantes en los últimos dos años u orales en los últimos seis meses. 
Las mujeres que cumplieron con estos criterios de selección entraron en un ensayo clínico controlado, abierto, comparativo y aleatorizado que buscaba conocer los efectos clínicos y las complicaciones de dos mezclas hormonales:

Grupo A, estrógenos conjugados $0.625 \mathrm{mg} /$ día por 21 días y acetato de medroxiprogesterona (MPA) $5 \mathrm{mg}$ del día 11 al 21, n: 49 mujeres.

Grupo B, valerato de estradiol $2 \mathrm{mg} /$ día y acetato de ciproterona (CPA) $1 \mathrm{mg}$ del día 11 al 21, preparado de investigación SHD 461, Schering AG, Alemania, n: 55 mujeres.

Ambos preparados fueron administrados a cada paciente durante un año, a partir de marzo de 1993; la última paciente culminó la fase clínica en noviembre de 1995.

El objetivo principal del estudio fue el de valorar el efecto de los dos preparados hormonales en la reducción de la frecuencia y la intensidad del síntoma vasomotor oleadas de calor, sin excluir la valoración de otros síntomas tales como sudoración, palpitaciones, trastornos del sueño y libido, entre otros.

Los componentes de los medicamentos fueron conocidos por el médico y la paciente. La asignación al tratamiento se realizó por medio de una lista de aleatorización pre-establecida, de acuerdo con la numeración de los estuches de los medicamentos del estudio, procedimiento realizado por Schering A. G. en Alemania.

Se llevaron a cabo controles al comienzo del estudio, y al mes, tercer, sexto, noveno y doce meses de THS, durante los cuales las pacientes evaluaron, mediante interrogatorio dirigido y con la ayuda de un diario, la intensidad y la frecuencia de los síntomas relacionados con el hipoestrogenismo; además, se realizó un examen físico general en cada visita. La información fue registrada en un formulario codificado con este fin (CRF).

Aparte de las recomendaciones generales sobre dieta y ejercicio durante esta fase de la vida de la mujer, no se dieron otras indicaciones especiales.

\section{Análisis estadístico}

Para el análisis se creó una base de datos en dbase y para el tratamiento de éstos se utilizó el programa EPIINFO 6.01 (CDC, 1994).

Se evaluaron las diferencias por grupo de tratamiento $y$ en los diferentes tiempos. Para las variables cuantitativas se realizó el análisis de ANOVA y para las cualitativas el test de chi cuadrado o el de Fisher, usando como nivel de significancia una $\mathrm{p}<0.05$.

\section{Resultados}

82 mujeres (grupo A: 38, grupo B: 44) completaron el período de 12 meses de observación. Se incluyen además en el presente análisis 6 mujeres que completaron por 10 menos 3 ciclos de tratamiento, para un total de 87 mujeres (Grupo A: 40, grupo B: 47), quienes cumplieron un total de 1.005 ciclos de tratamiento (grupo A: 462, grupo B: 543 ciclos).

La edad de la población estudiada fue de $50.8 \pm 4.5$, con un rango de edad entre 41 y 60 años; para el grupo A la edad fue de $51.3 \pm 4.8$ años, y para el grupo B $50.4 \pm$ 4.3. En ambos grupos predominó la raza mestiza, (grupo A: $80 \%$, grupo B: $74.5 \%$ ), seguida de la raza caucásica, con 17.5 y $25.5 \%$ respectivamente.

Con respecto al nivel socio-económico, predominó el nivel bajo (50 y $42.5 \%$ ) para los grupos A y B respectivamente y el nivel medio con 37.5 y $36.2 \%$ respectivamente. No hubo diferencias significativas en estas variables.

$72.5 \%$ de las mujeres del grupo A y $56.5 \%$ del grupo $B$ estaban en menopausia (amenorrea ${ }^{3} 12$ meses). Para el grupo A la amenorrea tenía una duración de $36.3 \pm 35.9$ meses (mediana $=24.0$ meses) y para el grupo B $26.8 \pm$ 30.9 (mediana $=15.5$ meses) al iniciar el tratamiento. No hubo diferencia significativa en la duración de la amenorrea para los dos grupos $(p=0.19)$.

Causas de abandono: Un total de 20 pacientes (11 del grupo A y 9 del grupo B) no completaron los 12 meses de terapia de THS. 14 se retiraron antes del tercer mes, 6 entre los tres y doce meses.

Los motivos no médicos de abandono (14 pacientes) fueron: Incumplimiento con el protocolo por no asistencia a los controles (4), viajes (2), temor al cáncer (1), deseo de alternativa homeopática (1) y causa desconocida (6).

Las causas de abandono por motivo médico (6 pacientes) se dividieron en aquellas probablemente relacionadas con la THS (4) y aquellas de presentación concomitante con la misma, pero no casualmente relacionadas (2).

Un caso correspondió al grupo A (depresión) y tres al grupo B (reaparición de asma con la THS, mastalgia y hemorragia genital respectivamente).

Se presentó un caso de hepatitis viral (no clasificada), de aparición simultánea con el comienzo de la THS (grupo A) y que obligó a su suspensión y una hemorragia genital el primer día del primer ciclo de la THS en otra paciente del grupo B. No se consideró la existencia de causalidad entre la THS y la aparición de estas alteraciones.

Es de considerar que durante el transcurso del estudio no se presentaron efectos adversos severos tales como enfermedad tromboembólica venosa, eventos coronarios ni enfermedad cerebrovascular.

Efectos secundarios: Los efectos secundarios más frecuentes en ambos grupos fueron: Tensión mamaria, náusea, edema y cefalea en orden decreciente. En general, no se observaron diferencias importantes de estos efectos secundarios entre los grupos, a pesar que en el primer y tercer ciclos se observó una mayor proporción de pacientes con tensión mamaria en el grupo B, no siendo esta diferencia estadísticamente significativa. En menor proporción se observaron otros efectos adversos como mareo, cansancio y dismenorrea, entre otros. En la mayoría de los casos, estos efectos secundarios fueron transitorios y se presentaron más frecuentemente entre el primer y tercer mes de la terapia, para disminuir progresivamente al término de un año (Tabla 1). 
Tabla 1

\section{FRECUENCIA DE EFECTOS SECUNDARIOS EN MUJERES BAJO THS* DURANTE UN AÑO}

\begin{tabular}{|c|c|c|c|c|c|c|c|c|c|c|}
\hline \multirow[t]{2}{*}{ Ciclo* } & \multicolumn{2}{|c|}{ Grupo } & \multicolumn{2}{|c|}{$\begin{array}{l}\text { Tensión } \\
\text { Mamaria }\end{array}$} & \multicolumn{2}{|c|}{ Edemas } & \multicolumn{2}{|c|}{ Náusea } & \multicolumn{2}{|c|}{ Cefalea } \\
\hline & $A^{1}$ & $\mathrm{~B}^{2}$ & A & B & A & $B$ & A & B & A & B \\
\hline 1 & $n=40$ & $n=47$ & $17.5 \%$ & $31.9 \% "$ & $7.5 \%$ & $6.4 \%$ & $15.0 \%$ & $10.6 \%$ & $2.5 \%$ & $2.1 \%$ \\
\hline 3 & $n=40$ & $n=47$ & $20.0 \%$ & $34.0 \% \cdots$ & 0 & $6.4 \%$ & $2.5 \%$ & $4.2 \%$ & $2.5 \%$ & $2.1 \%$ \\
\hline 12 & $n=38$ & $n=44$ & $18.4 \%$ & $22.7 \%$ & $2.6 \%$ & $4.5 \%$ & $2.6 \%$ & $2.2 \%$ & 0 & $4.5 \%$ \\
\hline
\end{tabular}

Estrógenos conjugados / Acetato de medroxiprogesterona

Valerato de estradiol / Acetato de ciproterona

Numero de mujeres que completan uno, tres y doce ciclos (meses) de THS

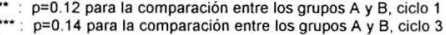

THS = Terapia Hormonal de Sustitución.

Es interesante anotar que en ambos grupos se observó en algunas mujeres la reaparición leve de síntomas como cefalea y oleadas de calor en la semana libre de tratamiento.

\section{Efecto de la THS sobre la intensidad y la frecuencia} de las oleadas de calor

La intensidad de las oleadas de calor se redujo en ambos grupos y desde el primer mes de tratamiento, de manera persistente y continuada (grupo $\mathrm{A}=75.5 \%$, grupo $\mathrm{B}=83.3 \%$ al cabo de un año, Tabla 2 ). La diferencia fue significativa dentro de cada uno de los grupos $(p<0.0001$ para el grupo A y $p<0.00001$ para el grupo B). Al comparar los resultados a los doce meses con la visita inicial, sin existir diferencia entre ambos compuestos entre sí. La mejoría observada fue mayor para aquellas mujeres quienes la intensidad fue evaluada inicialmente como severa.

La frecuencia de las oleadas de calor se redujo igualmente en ambos grupos a lo largo del tiempo, observándose el mayor efecto entre el primer y el tercer mes de tratamiento, y especialmente para aquellos episodios clasificados inicialmente como severos (más de cinco

\section{Tabla 2}

INTENSIDAD DE OLEADAS DE CALOR EN MUJERES BAJO THS ' DURANTE UN AÑO

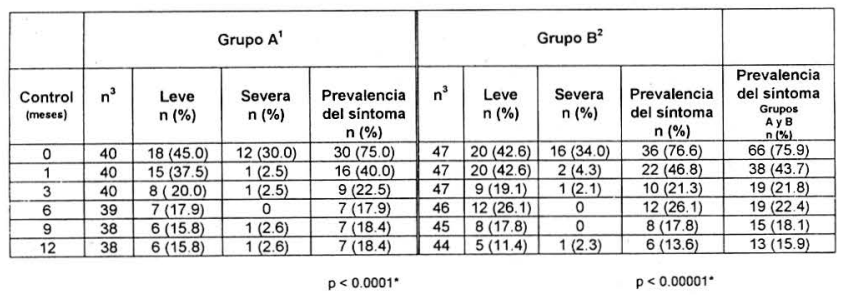

episodios por día, 26.4\%), los cuales desaparecieron prácticamente desde el tercer mes (Tabla 3). La frecuencia de los episodios leves se redujo de $49.4 \%$ al principio del estudio a sólo un $17.1 \%$ al final para la totalidad de las pacientes. Con respecto a los grupos $\mathrm{A} y \mathrm{~B}$, hubo diferencia estadisticamente significativa en el tiempo, entre el comienzo y el final $(\mathrm{p}<0.00001)$, pero no los grupos entre sí.

Un comportamiento similar se encontró con el síntoma de la sudoración nocturna, la cual se redujo progresivamente y de manera similar para ambos grupos, en particular, en aquellas mujeres quienes evaluaron la sudoración como severa. Al finalizar el estudio se encontró diferencia significativa para los dos grupos en el tiempo (grupo A, ciclo 1 vs. ciclo 12, $\mathrm{p}<0.0001$ y grupo $\mathrm{B}$, ciclo 1 vs. ciclo $12, \mathrm{p}<0.000001)$, pero no en 1 os grupos entre sí (Tabla 4).

Otro síntoma referido por las mujeres perimenopáusicas del presente estudio fue la sensación de palpitaciones, manifestación que se redujo en casi un 50\% desde el primer mes de tratamiento persistiendo con una frecuencia entre el 10 y el $16 \%$ para ambos grupos hasta el final del estudio. No hubo diferencia significativa entre los dos grupos ni en los diferentes tiempos.

Tabla 3

\section{FRECUENCIA DE OLEADAS DE CALOR EN MUJERES BAJO THS ' DURANTE UN AÑO}

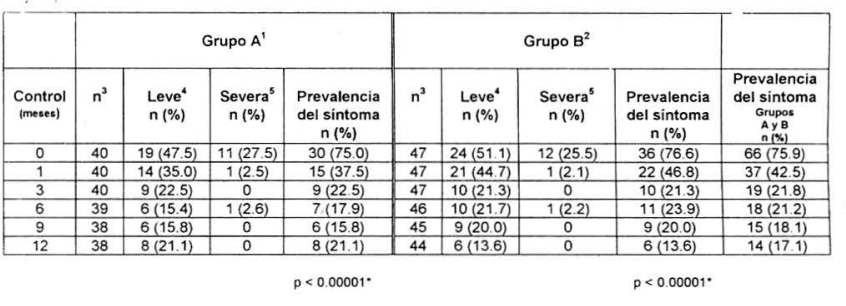

Estrógenos conjugados + acetato de medroxiprogesterona Valerato de estradiol + acetato de ciproterona Numero de pacientes que completan el respectivo control en cada grupo Más de cinco episodios de oleadas de calor por dia Maral a comparación entre la visita inicial y doce meses
THS = Terapia Hormonal de Sustitucion
Tabla 4

SUDORACION NOCTURNA EN MUJERES BAJO THS

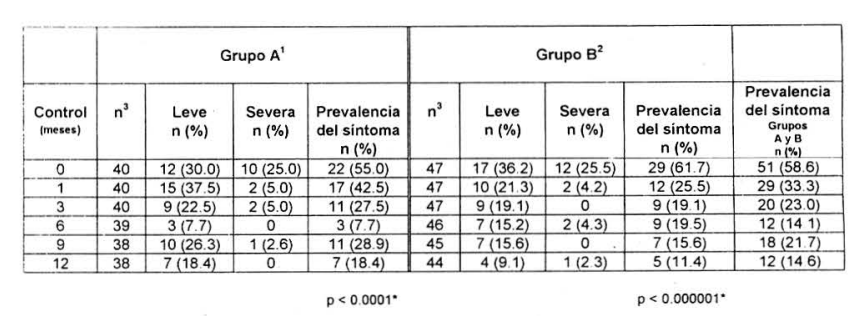

\section{DURANTE UN AÑO}

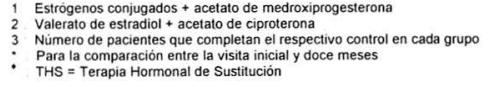




\section{Trastornos del sueño}

Se investigó sobre la presencia tanto de insomnio como de la fragmentación del sueño. Con respecto al insomnio, este síntoma fue referido inicialmente por la tercera parte de las mujeres en el grupo total, siendo más frecuente en el grupo B con relación al A. Esta manifestación se redujo en un $50 \%$ al cabo de un año para ambos grupos, sin alcanzar diferencia significativa. No hubo tampoco diferencia entre los dos grupos (Tabla 5).

Si se observó mejoría en la severidad del síntoma, pues el porcentaje de mujeres que calificó su insomnio como severo, pasó de $8 . \%$ a $1.2 \%$ al final del estudio.

La fragmentación del sueño fue referida por un $43 \%$ de las mujeres al comenzar el estudio y su frecuencia se redujo en un $45 \%$ después de un mes de tratamiento, alcanzando un $19.5 \%$ al cabo de un año. Se apreció además una disminución de la severidad del síntoma, pues se redujo la frecuencia de los trastornos severos de fragmentación del sueño de un $9.2 \%$ a $2.4 \%$ al final del estudio. La mejoría en la fragmentación del sueño fue significativa en el grupo B, a diferencia del grupo A (Tabla 6).

\section{Tabla 5 \\ INSOMNIO EN MUJERES BAJO THS' DURANTE UN AÑO}

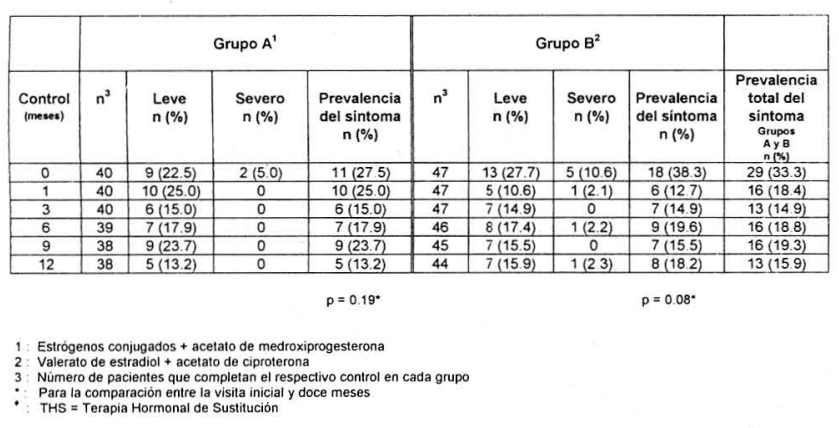

Tabla 6

\section{SUEÑO FRAGMENTADO EN MUJERES BAJO THS} DURANTE UN AÑO

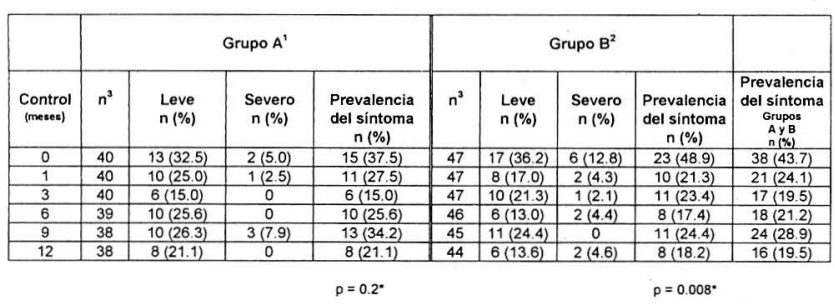

Estrogenos conjugados + acetato de medroxiprogesterona Valerato de estradiol I acelato de ciproterona
Número de pacientes que completan el respectivo control en cada grupo Numero de pacientes que completan el respectivo contro
Para la comparación entre la visita incial y doce meses
THS = Terapia Hormonal de Sustitución
Trastornos urinarios subjetivos como la incontinencia y la disuria no sufrieron modificaciones apreciables en ninguno de los dos grupos a lo largo del período de observación.

Se interrogó a las pacientes sobre trastornos de la sexualidad, en particular la presencia de dispareunia y alteraciones de la libido. Se excluyeron del análisis aquellas mujeres que no tenían vida sexual o quienes no dieron información durante el interrogatorio. Un $33.8 \%$ de 68 mujeres del estudio refirió la presencia de dispareunia, síntoma que mejoró en un $40 \%$ con el tratamiento hormonal, observándose la mejoría más importante desde el primer mes. Al final del estudio, sólo el $18.3 \%$ de las pacientes refería esta alteración.

Igualmente se observó una mejoría dramática con respecto a la severidad de la dispareunia, pues desapareció en las ocho mujeres que la habían calificado inicialmente como severa. No se apreció diferencia significativa entre los grupos ni en los diferentes tiempos.

La disminución del deseo sexual se presentó inicialmente en $41 \%$ de 71 mujeres del estudio, siendo más frecuente en el grupo A que en el B y no hubo mayores modificaciones ni en la frecuencia ni en la severidad de este síntoma a lo largo del estudio para los dos grupos.

\section{Trastornos psico-afectivos}

Las mujeres fueron interrogadas acerca de la presencia de cambios a nivel de su capacidad de concentración y la presencia de nerviosismo y depresión. Se encontró una alta frecuencia de mujeres que referían disminución en la capacidad de concentración (51\%) en ambos grupos al iniciar la THS. A lo largo del seguimiento las modificaciones observadas fueron muy variables en la prevalencia de este síntoma, pero lo que sí es notorio, fue la mejoría en la severidad del mismo que pasó de $9.2 \%$ a sólo un $2.4 \%$ al final del estudio.

Fue llamativo igualmente, que un porcentaje alto de mujeres $(62.1 \%)$ manifestaron la presencia de nerviosismo referido espontáneamente al comenzar el estudio, síntoma que descendió progresivamente a un $26.8 \%$ al final del tratamiento. Fue también notoria la disminución en la severidad del síntoma, percibido como severo por el $16,1 \%$ de las mujeres al iniciar la THS y por el $2.4 \%$ al finalizar la misma.

Se observó en ambos grupos una reducción significativa de la percepción de manifestaciones depresivas a lo largo del tiempo, pero no entre los grupos entre sí, estando presentes en el $46 \%$ de las mujeres que ingresaron al estudio. Al final del mismo, un $23 \%$ referían esta alteración.

No hubo modificaciones de importancia en la frecuencia ni en la severidad del dolor lumbar. Su frecuencia inicial en el grupo total fue de $41.4 \%$ y al final del estudio la tercera parte de las mujeres aún persistían con este síntoma, con un comportamiento muy similar para ambos grupos.

\section{Modificaciones del peso}

No se apreciaron cambios en el índice de masa corporal [peso en $\mathrm{Kg} / \mathrm{talla}^{2}(\mathrm{~m})$ ] al cabo de un año con respecto a los valores iniciales ni para el grupo A ni para el grupo B. Los valores obtenidos se encuentran dentro del rango normal (Figura 1). 
Figura 1

MODIFICACIONES DEL PESO MEDIDO POR EL INDICE DE MASA CORPORAL (IMC) EN 87 MUJERES EN MENOPAUSIA TRATADAS DURANTE UN AÑO CON TERAPIA HORMONAL DE SUSTITUCION CON DOS ESQUEMAS DIFERENTES

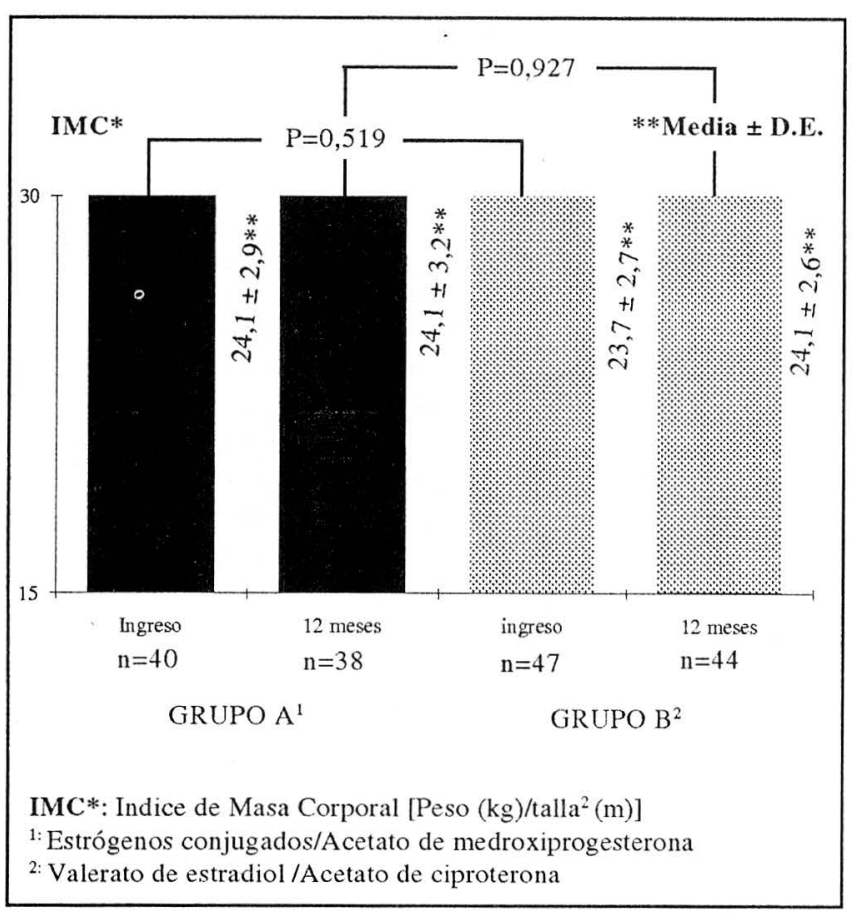

\section{Discusión}

Hasta un $70 \%$ de las mujeres en climaterio pueden presentar diversas manifestaciones psicosomáticas que reflejan un déficit estrogénico y en parte también progestacional. Entre las principales manifestaciones subjetivas carenciales de este período se distinguen principalmente las oleadas de calor, la sudoración nocturna y las alteraciones del sueño, así como manifestaciones de cansancio, nerviosismo, irritabilidad y de alteración en la capacidad de concentración. Todos estos fenómenos están comprendidos dentro de la sintomatología del climaterio, la cual puede afectar de manera adversa el estado general y la calidad de vida de las mujeres en este período de la vida (1).

El estudio comparativo de los dos preparados hormonales A y B se efectuó con un grupo de mujeres en perimenopausia sanas, seleccionadas y controladas, y con edades entre los 40 y 60 años. Las voluntarias estaban motivadas alrededor de los objetivos de la investigación, lo cual aseguraba la adherencia a los dos esquemas de THS.

El presente estudio confirmó la rápida e importante disminución en la frecuencia e intensidad de uno de los principales síntomas del climaterio como son las oleadas de calor, las cuales suelen aparecer precozmente desde meses antes de la amenorrea definitiva. Estas oleadas de calor fue el síntoma de presentación más frecuente en la presente investigación $(75,9 \%)$, corroborando los datos obtenidos en el estudio cooperativo sobre la epidemiología de la menopausia en Colombia (2), en donde se observó una frecuencia del $66 \%$. Las oleadas de calor suelen durar desde unos segundos a varios minutos, siendo varios los episodios durante el día y la noche los cuales pueden ocasionar en la mujer fragmentación del sueño, adinamia y fatigabilidad $(3,11)$.

Una vez se inicia la terapia hormonal (THS), la sintomatología mejora rápidamente. En el presente estudio, el síntoma de las oleadas de calor se redujo en un $45 \%$ al cabo de un mes y por lo menos en $70 \%$ a los tres meses para los dos grupos. Igual fenómeno se observó con la severidad del síntoma y con la frecuencia de las oleadas.

Es importante apreciar cómo ambos esquemas terapéuticos disminuyeron de manera igualmente eficaz los fenómenos anteriormente anotados, aunque siempre habrá un escaso porcentaje de pacientes en quienes los síntomas persisten, pero con menor intensidad y frecuencia. En estos casos puede ser útil la prescripción adicional de medicamentos que actúen como reguladores neurovegetativos.

Kuhi señala como la terapia de sustitución con estrógenos induce una notoria mejoría y aún la desaparición de las oleadas y la sudoración, en el $80 \%$ de las mujeres en un término de tres meses (1).

El mecanismo de acción de estas crisis vasomotoras aún no se ha dilucidado claramente y pareciera correlacionarse con el descenso de los niveles de estrógenos en el sistema nervioso central (SNC), lo cual desencadenaría un desequilibrio en el centro termorregulador como consecuencia de alteraciones en la concentración de neurotransmisores adrenérgicos, dopaminérgicos y opioides, asociadas a elevaciones de GnRH (11-12).

Dado que se han encontrado receptores nucleares específicos para estrógenos especialmente en hipófisis, hipotálamo y sistema límbico, se postula que los estrógenos ejercerían un papel modulador sobre el centro termorregulador mediante la estimulación de la síntesis de neurotransmisores. No obstante, otras sustancias también pueden ejercer esta función, como los progestágenos, los cuales fueron administrados simultáneamente a las mujeres de esta investigación; otras sustancias alfa adrenérgicas como la clonidina, antidopaminérgicas como el veralipride y antiopioides como el naloxano, complementarían la acción estrogénica sobre las oleadas de calor (13-15).

Las oleadas de calor suelen acompañarse de grados diversos de diaforesis diurna y nocturna que afectan adversamente el bienestar de la mujer (16). Su frecuencia inicial en el presente estudio (59\%) fue muy similar a la encontrada por Onatra y cols., de 52\% (2). En el presente estudio la THS redujo en cuatro veces esta manifestación y fue especialmente notoria la reducción en su severidad, lo cual tiende a mejorar la calidad de vida de la mujer.

Fue relativamente alto el porcentaje de mujeres que presentaron alteraciones del sueño. El $40 \%$ de ellas refirieron fragmentación del sueño y una tercera parte insomnio, situación similar a la informada en la literatura 
(2). Es evidente la mejoría observada en cuanto a la disminución en la fragmentación del sueño, no así con las manifestaciones de insomnio, que sólo se redujeron de manera muy discreta y sin significancia estadística. Sin embargo, es importante apreciar cómo si se redujo la severidad de este síntoma, por lo cual se puede aceptar que la THS juega un papel importante en la regulación del sueño (17), si bien es cierto que estos trastornos pueden tener otras causas etiológicas que deben tenerse en cuenta, tales como problemas personales, familiares, económicos, laborales, etc. (3).

Trastornos del sueño que se presentan durante la inducción (insomnio) o de fragmentación suelen ser las quejas más frecuentes de la mujer durante el climaterio, $\mathrm{y}$ provocan un descanso superficial que genera como consecuencia somnolencia diurna, adinamia, fatigabilidad y empeoramiento de manifestaciones psicoafectivas previas.

La frecuencia de síntomas urinarios manifestados por incontinencia $(15 \%)$ y disuria $(2 \%$ a $8 \%)$ que se presentó en esta población fue relativamente baja y es similar al porcentaje informado por la literatura. No hubo modificación importante con la THS, lo que sugiere que en el grupo de mujeres analizadas, y por las características de la población encontradas (primeros años de la menopausia), esta sintomatología puede ser secundaria a la relajación del piso pélvico, infecciones urinarias o vulvovaginales asociadas, a diferencia de mujeres con edad más avanzada en donde el hipoestrogenismo desencadena atrofia del piso pélvico y el árbol urinario, y como consecuencia provoca incontinencia y urgencia urinaria (1-4).

EI déficit estrogénico prolongado puede ocasionar atrofia progresiva del epitelio uretral y vesical, a lo cual se suman los cambios degenerativos del tejido conectivo mediados por la destrucción del colágeno tipo 1 en especial alrededor de la uretra y del piso pélvico, la disminución de la irrigación tisular y alteraciones en la integridad del sistema neuromuscular. Aproximadamente un $25 \%$ de las mujeres con edades entre los 40 y 50 años sufren de incontinencia y este porcentaje se eleva con la edad progresiva (1, 3-4).

La terapia de sustitución con estrógenos ocasiona una manifiesta mejoría de los cambios atróficos del epitelio urogenital y de los tres principales síntomas urinarios (disuria, frecuencia y urgencia), ya que ocasiona una proliferación regenerativa de los epitelios por mayor síntesis de colágeno, aumenta la elasticidad de la uretra, mejora el tono de la musculatura pélvica e induce una mayor irrigación y transudación y un aumento importante del contenido colágeno y de agua de los tejidos; igualmente se observa una normalización del pH local $(1,4,18-22)$.

\section{Trastornos de la sexualidad}

Es conocido que la sexualidad durante la menopausia se puede alterar por causas hormonales, orgánicas y psicológicas. Como consecuencia del hipoestrogenismo prolongado, pueden ocurrir modificaciones en el tracto genital, en particular, atrofia del epitelio vaginal y acortamiento de la vagina a expensas de estenosis del tercio superior con paredes lisas y estrechas. A nivel vulvar, hay pérdida de la grasa y elasticidad de los tejidos y como resultado de ello, estrechez del introito, atrofia del clítoris y de las glándulas de Bartolino; disminución de la irrigación sanguínea, y por ende se reducen la transudación y la lubricación. Las pacientes se quejan de sequedad vaginal, ardor, prurito y dispareunia. Por la reducción en la síntesis de glucógeno y la disminución de las secreciones vaginales, se eleva el $\mathrm{pH}$ con alteración de la flora y desaparición de los lactobacilos, todo lo cual facilita la aparición de infecciones locales. Igualmente hay atrofia del cuello y del útero como también del tejido fibromuscular y de los ligamentos de sostén del piso pélvico. La vascularización pélvica también se reduce (1, 3-5, 12, 18, 21-23).

Es reconocido el benéfico efecto que ejercen los estrógenos sobre estas manifestaciones carenciales, debido a su efecto proliferativo del epitelio, la mejoría de la irrigación y el aumento en el contenido de agua y colágeno en los tejidos, normalización simultánea de las propiedades físico-químicas y microbiológicas de la vagina (1).

La dispareunia fue un síntoma que se presentó en un porcentaje relativamente alto en mujeres con vida sexual activa, $34.3 \%$, esto es, una de cada tres mujeres en el presente estudio, lo cual constituye una frecuencia mayor a la observada en el estudio cooperativo colombiano $(13 \%)$. Con la THS se logró una mejoría importante hasta alcanzar una reducción del $50 \%$ al cabo de un año, que si bien no fue estadísticamente significativa, si refleja muy probablemente la acción hormonal a nivel genital. La persistencia de este síntoma al final del estudio, cercana al $20 \%$ para ambos grupos, continúa siendo alta como consecuencia de otros factores no determinados que inciden en su presentación.

Las alteraciones de la libido se presentaron con una frecuencia alta del $44.7 \%$, para la totalidad de las voluntarias incluidas, lo que en la práctica significa que una de cada dos mujeres tiene una alteración en esta área, aspecto por lo demás preocupante. Es llamativo que este síntoma no presentó mejoría con la THS, por el contrario en algunas mujeres tendió a agravarse a pesar de la disminución en la frecuencia de la dispareunia, lo cual sugiere que existen otras causas tales como problemas psicológicos, de pareja, familiares, económicos, laborales, sociales y eventualmente el déficit androgénico relativo, sobre los cuales la terapia estro-gestágena no ejerce un efecto directo.

\section{Trastornos psico-afectivos}

Los trastornos psicológicos durante el climaterio se pueden dividir en cinco grupos: 1. Disminución de la concentración y la memoria, 2. Astenia y adinamia, 3. Nerviosismo, excitación y ansiedad, 4. Depresión, y 5. Disminución o pérdida de la libido y frigidez (23).

Estos trastornos rara vez requieren tratamiento psiquiátrico, puesto que el climaterio, en sentido amplio, cursa con alteraciones psicológicas que afectan el estado de ánimo y que podrían calificarse como depresión reactiva leve. No obstante, un $15 \%$ a $20 \%$ de esta población muestra una asociación clara entre menopausia, 
trastornos psicológicos y consulta médica, en quienes la THS y la psicoterapia que efectúe el médico pueden ser básicos para la recuperación de la auto-estima de la paciente.

A los estrógenos se le han atribuido varios mecanismos de acción sobre el sistema nervioso central, entre los cuales se destacan el aumento de la concentración de triptofano libre necesario para la síntesis de catecolominas, una mayor degradación de la monoaminooxidasa (MAO) con menor degradación y aumento en la concentración de neurotransmisores tales como noradrenalina, serotonina y dopamina, un mayor transporte de serotonina al SNC y finalmente, una acción neurotrópica directa sobre el desarrollo neuronal y de las conexiones sinápticas. Como consecuencia de lo anterior, se han descrito diversos y variados efectos de los estrógenos sobre las funciones afectivas y cognoscitivas (24-30).

En orden de frecuencia las principales manifestaciones psico-afectivas encontradas en el presente estudio fueron nerviosismo $(61,6 \%)$, disminución de la concentración $(50 \%)$ y depresión $(46,5 \%)$, a diferencia del estudio nacional (2) donde se encontró una frecuencia de manifestaciones depresivas del $20 \%$. Alrededor de la mitad de las mujeres incluidas en este estudio, presentaron entonces manifestaciones psicoafectivas, situación que de por sí incide en la calidad de vida y en la autoestima de la mujer. Con la THS hubo una reducción significativa con respecto al tiempo pero no en los diferentes grupos entre sí en lo relacionado con la percepción de manifestaciones depresivas, cercana al $50 \%$. Con respecto a las alteraciones en la capacidad de concentración y la presencia de nerviosismo, el principal cambio observado fue reducción en la severidad de estos síntomas manifestados espontáneamente, si bien deben tenerse en cuenta las limitaciones del presente estudio en la evaluación de estos síntomas ya que su presencia se estableció por interrogatorio simple sin aplicar pruebas especiales para su diagnóstico.

Por otra parte, las pacientes fueron interrogadas acerca de la presencia de lumbalgia y se estableció que este fue un síntoma relativamente frecuente al comienzo del estudio $(41.9 \%)$, esto es, prácticamente una de cada dos mujeres, a diferencia del estudio cooperativo en donde sólo alcanzó el $18 \%$. Con la THS se produjo una reducción de sólo un $10 \%$ en ambos grupos de tratamiento, no significativo; al final del estudio una de cada tres mujeres persistía con esta sintomatología, y tampoco se observaron modificaciones de importancia en cuanto a la intensidad del síntoma referido como severo en $8.1 \%$ de las mujeres al comienzo del estudio y en $7.3 \%$ al final. Este hallazgo muy seguramente refleja la etiología multifactorial de este síntoma, no solamente causado por osteoporosis (31-32), sino como expresión de múltiples patologías a nivel osteomuscular o referidas por alteraciones en órganos abdominopélvicos, sin olvidar las causas psicógenas. La mayoría de estas alteraciones no son susceptibles de mejorar con la THS.

Finalmente se hizo un seguimiento del comportamiento del peso corporal bajo el tratamiento de sustitu- ción hormonal y no se observó ninguna variación en el índice de masa corporal al cabo de un año en ninguno de los dos grupos. Es de resaltar que las mujeres no recibieron instrucciones especiales sobre ningún tipo de dieta en particular (33) y por ende, el presente estudio puede reflejar la situación de la práctica clínica diaria y permite al médico asegurar a sus pacientes que no cabe esperar modificaciones importantes en el peso corporal bajo la THS a corto y largo plazo, de acuerdo con lo reportado en la literatura. Se debe recordar que una de las principales causas de abandono de la THS informadas en la literatura, es el infundado temor a subir de peso, toda vez que muchas mujeres son reacias a la THS, pues atribuyen el aumento de peso a la misma. (34-35).

Riis pudo demostrar igualmente estabilidad del peso en mujeres tratadas durante dos años con valerato de estradiol y acetato de ciproterona, con respecto al grupo control, el cual presentó un incremento de $2 \mathrm{~kg}$ (32). Una respuesta similar fue informada por Hassager al utilizar durante dos años valerato de estradiol, acetato de ciproterona y estradiol percutáneo y dos grupos control, uno oral y otro transdérmico. Se apreció estabilidad de peso con relación a los grupos control, cuyo aumento de peso fue de 1 a $2 \mathrm{~kg}$ en 24 meses (36).

Se ha postulado entonces que los factores causales del aumento de peso durante la postmenopausia pueden estar más bien relacionados a una mayor ingesta calórica en presencia de un menor requerimiento para la edad y un mayor sedentarismo con menor gasto energético (35). Otro factor postulado lo constituye la pérdida de estrógenos endógenos con redistribución de la grasa corporal a expensas de aumento de la grasa abdominal, alteración de la relación cintura-cadera $(>0.84)$, reabsorción del colágeno tipo 1 y 2 y disminución de la masa osteomuscular $(32,36)$.

En un estudio de seguimiento efectuado en 1.286 mujeres postmenopáusicas durante 15 años (34) y después de ajustar por edad e IMC basal, no se encontró diferencia significativa entre usuarias y no usuarias de estrógenos en lo que respecta a medidas de obesidad, composición o distribución de grasa corporal. Al ajustar para el uso de gestágenos, tampoco variaron los resultados. Los autores concluyen que la THS no tiene ninguna relación con la ganancia de peso, la obesidad o la distribución central de grasa en mujeres postmenopáusicas.

Un reciente estudio longitudinal controlado realizado en Baltimore, U.S.A., analizó los efectos de la deficiencia hormonal postmenopáusica sobre el balance energético y la composición corporal. Se encontró en estas mujeres una pérdida de masa corporal magra, una ganancia de tejido graso y una disminución del metabolismo energético basal de aproximadamente $100 \mathrm{kcal} /$ día, con una disminución del gasto energético por mayor sedentarismo, de $130 \mathrm{kcal} /$ día, una ingesta calórica invariable y un aumento en los niveles de insulina. Aparte de los riesgos cardiovasculares inherentes a estos cambios, los autores demuestran que la ganancia de peso es resultado de la deficiencia estrogénica y no de la THS y debida básicamente a alteraciones del balance energético (35). 


\section{Conclusiones}

En resumen, el presente estudio demuestra el efecto benéfico de la THS sobre los principales síntomas en el climaterio en mujeres sanas en perimenopausia sanas. En términos generales, se observó una rápida disminución de los síntomas del climaterio (oleadas de calor, sudoración nocturna) entre el primer y tercer mes de THS, tanto en su frecuencia como en su intensidad. Es importante apreciar, sin embargo, que algunos síntomas (dispareunia, alteraciones en la capacidad de concentración, nerviosismo, manifestaciones depresivas) persistieron hasta el final del período de observación pero con atenuación de su severidad, haciéndose más tolerables. En general, la tolerancia a los dos preparados hormonales fue buena y los abandonos directamente ocasionados por la THS fueron pocos. La mayor parte de los efectos secundarios fue de carácter transitorio y tuvo su mayor ocurrencia entre el primer y tercer mes de la THS, para disminuir progresivamente. No se presentaron complicaciones tromboembólicas. Para algunos síntomas, las diferencias estadísticas encontradas entre los dos grupos no tienen significado clínico.

Ambos preparados hormonales demostraron ser igualmente eficaces, con comportamientos semejantes a los informados por la Iiteratura (37) y sin que se pudieran demostrar diferencias significativas entre sí.

Si bien, para el presente estudio se seleccionaron mujeres sanas, motivadas y con una alta percepción de su sintomatología, los resultados obtenidos tienen validez para la población general, toda vez que las pacientes reclutadas fueron exhaustivamente seleccionadas y cumplen con el perfil de la mujer sana que requiere tratamiento por sus molestias del climaterio. Hemminki se ha referido ampliamente a las características especiales de las mujeres que son incluidas en estudios de THS (38).

A pesar que la THS es una herramienta muy útil, muchos síntomas que la mujer presenta durante la menopausia son de origen multifactorial y no desaparecen totalmente, debido a la existencia de otros fenómenos desencadenantes de orden personal, familiar, y del entorno sociocultural de la mujer climatérica, lo que hace que la THS por sí sola no sea suficiente para mejorar estos síntomas $(4,39)$. También debe tenerse en cuenta que la especialidad del médico tratante y sus conocimientos y actitud acerca de la THS son factores muy importantes que inciden sobre el manejo de la mujer en menopausia y postmenopausia (40). Se hace necesario entonces que el médico, además de conocer las diversas modalidades de THS, escuche, oriente y motive a la mujer en esta etapa de la vida.

\section{Agradecimientos}

A Angela Obregón y Stella Pardo por su ayuda en la transcripción del manuscrito.

\section{BIBLIOGRAFIA}

1. Kuhl H. Substitutionstherapie mit Oestrogenen und Gestagenen. Dtsch Med Wschr 1995; 120: 765-768.

2. Onatra W., Sánchez J., Acuña G. Epidemiología de la menopausia en Colombia: estudio cooperativo. Rev. Colomb. Obstet. Ginecol. 1994; 45S: 20-27.

3. Brenner P. The menopausal syndrome. Obstet. Gynecol. 1988; 72: 6S-11S.

4. Belchetz PE. Hormonal treatment of postmenopausal women. N. Engl. J. Med. 1994; 330: 1062-1071.

5. Ettinger B. Optimal use of postmenopausal hormone replacement. Obstet. Gynecol. 1988; 72: 31S-36S.

6. Sitruk-Ware R. Estrogen therapy during menopause. Practical treatment recommendations. Drugs 1990; 39: 203-217.

7. Udoff L., Langenberg P., Adashi E. Combined continuous hormone replacement therapy: A critical review. Obstet. Gynecol. 1995; 86: 306-316.

8. Sánchez F. Terapia de sustitución hormonal. Rev. Colomb. Obstet. Ginecol. 1994; 45: 10S-19S.

9. European Menopause Society. European Consensus Development Conference on Menopause. Human. Reprod. 1996; 11: 975-979.

10. Dirección de Recursos Humanos. Ministerio de Salud. República de Colombia. Conferencia de Consenso sobre Terapia de Suplencia Hormonal en la Menopausia, conclusiones y recomendaciones. Rev. Colomb. Menopausia 1996; 2: 46-49.

11. Tulandi T., Lal S. Menopausal hot flush. Obstet. Gynecol. Surv. 1985; 40: 553-563.

12. Hammond C. Menopause and hormone replacement therapy: an overview. Obstet. Gynecol. 1996; 87: 2S-16S.

13. Celades M. Aspectos clínicos en la menopausia. En: Palacios $\mathrm{S}$, ed. Climaterio y Menopausia. Madrid: Mirpal, 1993; 64-68.

14. Loprinzi CL., Michalak JC., Quella SK et al. Megestrol acetate for the prevention on hot flashes. N. Engl. J. Med. 1994; 331: 347-352.

15. Tulandi T., Lal S., Guyda H. Effect of estrogen on the growth hormone response to the alfa adrenergic agonist clonidine in women with menopause flushing. J. Clinic. Endocrinol. Metab. 1987; 65: 6-10.
16. Oldenhave A., Jaszmann LJB., Haspels AA. Impact of climacteric on wellbeing. Am. J. Obstet. Gynecol. 1993; 168: 772-780.

17. Schift I., Regestein Q., Tufchinsky D., Ryan KH. Efectos de los estrógenos sobre el sueño y el estado psicológico de las mujeres con el hipogonadismo. JAMA (Colombia) 1980; 3: 110-113.

18. Rodríguez KA., Onatra W. La menopausia: un estado fisiológico complejo. Acta Med. Colomb. 1993; 18: 257-267.

19. Sultana CJ., Walters MD. Estrogen and urinary incontinence in women. Maturitas 1995; 20: 129-138.

20. Castelo-Blanco C., Duran M., Gonzalez-Merlo J. Skin collagen changes related to age and hormone replacement therapy. Maturitas 1992; 15: 113-119.

21. Carafin L. Urologic problems. En: Eskin BA. The menopause, comprehensive management: New York: Mc Graw Hill 1994; 171181.

22. Palacios S., Menéndez C. Alteraciones del tracto genito-urinario bajo y menopausia. En: Palacios S, ed. Climaterio y Menopausia. Madrid: Mirpal, 1993; 105-112.

23. Barber H. Gynecologic problems. En: Eskin BA. The menopause, comprehensive management. New York: Mc Graw Hill 1994; $183-$ 210.

24. Salvatierra V. Alteraciones psicológicas y sexuales durante la menopausia. En: Palacios S, ed. Climaterio y menopausia. Madrid: Mirpal, 1993; 69-104.

25. Sherwin $B$. The impact of diffefent doses of estrogen and progestin on mood and sexual behavior in postmenopausal women. J. Clin. Endocrinol. Metab. 1991; 72: 336-343.

26. Ditkoff EC., Crary WG., Cristo M., Lobo RA. Estrogen improves psychological function in asymptomatic postmenopausal women. Obstet. Gynecol. 1991; 78: 991-995.

27. Schmidt PJ., Rubinow DR. Menopause-related affective disorders: a justification for further study. Am. J. Psychiatry. 1991; 148: 844-852.

28. Lindheim SR., Legro RS., Bernstein L. Behavioral stress responses in premenopausal and postmenopausal women and the effects of estrogen. Am. J. Obstet. Gynecol. 1992; 167: 1831-1836. 
29. Bevvley S., Bewley TH. Drug dependence with oestrogen replacement therapy. Lancet 1992; 339: 290-291.

30. Sherwin B. Hormones mood and cognitive functioning in postmenopausal women. Obstet. Gynecol. 1996; 87: 20S-26S.

31. Johnston CC. Osteoporosis. In : Eskin BA, ed. The menopause, comprensive management. New York: Mc Graw Hill, 1994; 103-115.

32. Riis BJ., Jensen J., Christiansen C. Cyproterone acetate, an alternative gestagen in postmenopausal oestrogen/gestagen therapy. Clinic. Endocrinol 1987; 26: 327-334.

33. Sutnick MR. Nutritional aspects of the menopause. In : Eskin BA, ed. The menopause, comprensive management. New York: Mc Graw Hill, 1994; 155-159.

34. Kritz-Silverstein D., Barret Connor E. Long term postmenopausal hormone use, obesity and fat distribution in older women. JAMA 1996; 275: 46-49.

35. Poehlman ET., Toth MJ. Changes in energy balance and body composition at menopause: A controlled longitudinal study. Ann. Intern. Med. 1995; 123: 673-675.
36. Hassager C., Christiansen C. Estrogen/gestagen therapy changes soft tissue body composition in postmenopausal women. Metabolism 1989; 38: 662-665.

37. Egarter C., Geurts P., Boschitsch E. The effects of estradiol valerate plus medroxyprogesterone acetate and conjugated estrogen plus medrogestone on climacteric symptoms and metabolic variables in perimenopausal women. Act. Obstet. Gynec. Scand. 1996; 75: 386-393.

38. Hemminki E., Malin M., Topo P. Selection to menopausal therapy by women's characteristics. J. Clin. Epidemiol. 1993; 46: 211219.

39. Alvarez R. El proceso de la familia en torno a la menopausia de la mujer. Rev. Colomb. Obstet. Ginecol. 1994; 45: 41S-45S.

40. Hemminki E., Topo P., Malin M., Kangas I. Physicians' views on hormone therapy around and after menopause. Maturitas 1993; 16: 163-173. 